\title{
Voice and swallowing implications in patients with tumors in their mediastinum
}

Elaine Cristina Bezerra dos Santos ${ }^{(1)}$ Danielle de Sousa Terto Jacob Diniz ${ }^{(1)}$ Amanda Roselle Cândido Correia(1) Roberta Borba Assis ${ }^{(1)}$

(1) Hospital de Câncer de Pernambuco, Recife, Pernambuco, Brasil.

Conflict of interests: Nonexistent

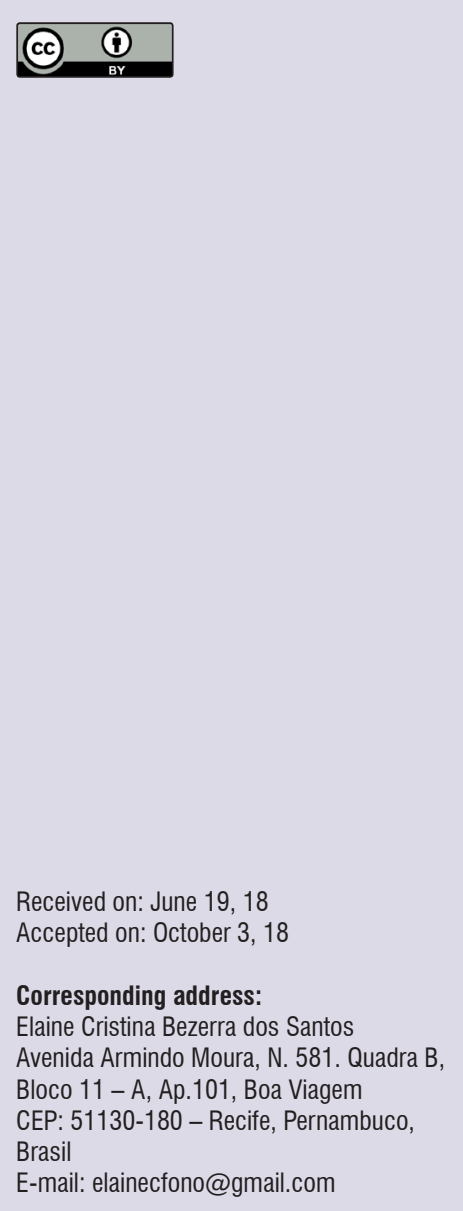

\section{ABSTRACT}

Purpose: to verify voice and swallowing implications in patients diagnosed with tumors in the mediastinum.

Methods: the study was carried out with 21 individuals aged between 18 and 60 years, with a diagnosis of tumors in their mediastinum. Data collection was performed at Oncology, OncoHematology and Thoracic Oncology Surgery ambulatory, and in the wards of the aforementioned clinics at an oncology reference hospital. The data was obtained by applying a questionnaire, and by evaluating voice and swallowing, using CAPE-V and O'Neil protocols, respectively.

Results: there was a higher prevalence of females with mean age at 40 years. A higher prevalence of tumors in the anterior region of the mediastinum and non-Hodgkin's lymphomas was found, however, Hodgkin's lymphomas presented worse results in all the parameters of the voice evaluation. Fourteen subjects presented some degree of dysphagia, ranging from mild to moderate.

Conclusion: patients with tumors in the mediastinum have significant impairments in voice and swallowing functions, especially when they are located in the anterior mediastinal region and Hodgkin lymphomas.

Keywords: Voice; Swallowing; Mediastinum Neoplasms; Speech, Language and Hearing Therapy 


\section{INTRODUCTION}

Malignant mediastinal tumors are rare $(75 \%$ of cases are benign), often asymptomatic and occur predominantly in adults ${ }^{1}$. According to Oliveira, Oliveira and Pietro ${ }^{2}$, the mediastinal masses include a great variety of tumors. These tumors may be congenital or acquired, of primary or secondary origin. The most common masses in the mediastinum include thymoma, lymphoma, pheochromocytoma, germ cell tumors and parathyroid lesions.

The space of the mediastinum is narrow and any mass in this region can compress adjacent structures leading to emergencies that threaten life ${ }^{3}$. The most frequent signs and symptoms involve respiratory changes, chest pain, weight loss and neurological disorders ${ }^{1}$. Other signs and symptoms that may have different impacts on the quality of life of subjects with mediastinal tumors are esophageal compression dysphagia, superior vena cava syndrome, invasion of the pericardium causing tamponade, and bronchial compression dysphonia ${ }^{4-6}$.

However, the appearance of these symptoms will depend on the histological type of the disease, the location of the tumor and the degree of nerve involvement ${ }^{7,8}$. In this study, the research on voice and swallowing functions was delimited in individuals with tumors in the mediastinum.

Dysphonia is characterized as any disturbance in vocal production that may present symptoms of different degrees, originated or aggravated by environmental, behavioral and / or traumatic risk factors ${ }^{9}$. In addition to functionally compromising vocal quality, changes in the laryngeal mechanism may result in lower airway (LAW) risks, since the larynx assumes the important protection function of LAW, respiration and phonation respectively.

In view of this, changes in laryngeal mobility may develop changes in safe swallowing, known as dysphagia, being associated with a high risk of mortality, if not diagnosed early9,10.

Dysphagia is a swallowing disorder involving a complex activity of voluntary and involuntary coordinated actions including neuromuscular structures of the oropharyngeal and esophagus. This alteration may result in food entering the airway, presenting as coughing, suffocation/asphyxia, pulmonary problems and aspiration ${ }^{11,12}$.

Therefore, the objective of this study was to verify the implications on voice and swallowing in patients with tumors located in the mediastinum.

\section{METHODS}

A cross-sectional, observational study, consisting of 21 individuals aged 18 to 60 years, with diagnosis of tumors in the mediastinum at a Reference Hospital in Oncology in the state of Pernambuco, approved by the Research Ethics Committee of the Pernambuco Society for Combating Cancer under No. 1.443.356. All participants signed the Informed Consent Form prior to the start of data collection.

The research is of characterization and consisted of two stages: 1.Investigation of the subjects through the Hospital Registry of Cancer of the referred institution; 2. Direct collection during consultations in the Clinical Oncology, OncoHematology and Oncologic Thoracic Surgery clinics, according to the eligibility criteria of the study.

Individuals of both sexes, aged between 18 and 60 years and diagnosed with tumors in the mediastinum, participated in the study. The exclusion criteria were: subjects with diagnosis of tumors involving oral cavity, oropharynx, larynx, thyroid, esophagus and lung, concomitantly to the tumor in the mediastinum; patients submitted to radiotherapy in the head and neck region; individuals with neurological impairment and tracheostomized patients.

The research consisted initially of filling out a specific questionnaire prepared for the study, consisting of identification and clinical data, such as tumor type, mediastinal location and oncological treatment performed, followed by voice and swallowing by means of protocols specific.

For voice recording, the portable digital recorder was used and for voice evaluation the CAPE-V (Auditory-Perceptual Evaluation of voice) scale was used, adapted to Portuguese in $2004^{13}$. This protocol for the perceptual-auditory assessment of the voice is composed of parameters that subjectively evaluate the general degree of voice deviation, roughness, breathiness, tension, pitch, loudness and resonance, contemplating a current tendency when using the analog-visual scale of $100 \mathrm{~mm}$ in the classification of these parameters ${ }^{13,14}$. The analysis of the data will be composed of the weighted arithmetic mean between the different groups studied, being diagnosis, tumor type, lymphoma type and tumor localization in the mediastinum.

In the evaluation, a recorder was used, where the participant was instructed to remain seated, and then asked the subject to send the sustained vowels / a / and / $\mathrm{i} /$ for three to five seconds; Besides the reading 
of six predetermined phrases: Érica drank some juice of pear and blackberry; Sonia knows how to samba alone; Look at the blue plane; Now it is time to finish; My mother dated an angel; and, Dad brought hot popcorn. And snippets of spontaneous speech. The evaluation of these parameters was computed in the CAPE-V14 from the listening of two evaluators.

For the functional evaluation of swallowing, the dysphagia severity scale (O'Neil) ${ }^{15}$ was used, although this tool was developed for objective evaluation of swallowing, due to the absence of other instruments, this has been presented as an excellent and complete among the instruments available for studies. In addition, this scale is considered as a valuable tool with high reliability to measure the efficacy of treatment of dysphagic patients. It is possible to classify deglutition between normal and severe oropharyngeal dysphagia, from level I to level VII, respectively.

For functional evaluation, different consistencies were considered, and the following foods and volumes were standardized: pasty $(50 \mathrm{ml}$ of water associated with the thickener food thickener); solid (one whole wafer) and liquid (50 $\mathrm{ml}$ water). During the evaluation, the subjects remained seated, indicating signs of changes in swallowing, such as coughs, throat coughs, sneezing, increased respiratory rate, change in vocal quality and alteration in cervical auscultation. In the non-invasive cervical auscultation, a stethoscope was used, which was positioned at the lateral border of the trachea, immediately inferior to the cricoid cartilage, assisting in the occurrence of laryngeal aspirations and penetrations. After the evaluation, the parameters were classified in the O'Neil scale ${ }^{15}$.

For data analysis, a database was built in the Microsoft Excel worksheet which was exported to the SPSS software. The normality of the score was assessed using the Kolmogorov-Smirnov test and, in cases where normality was indicated, the student's $t$ test and the ANOVA test were applied to compare the mean score of the parameters. In cases in which the normality of the scores of the evaluated parameters was not indicated, the Mann-whitney test and the Kruskal-Wallis test were applied. All conclusions were drawn considering the level of significance of $5 \%$.

\section{RESULTS}

The sample consisted of twenty-one subjects, where 11 were females and older than 40 years. It is observed that, on average, the patients had ages of 40.1 years with a standard deviation of 11.7 years (Table 1).

As seen in Table 1, 16 subjects had a primary tumor located in the mediastinum, with no evidence of disease elsewhere. Lymphoma presented a prevalence of eleven cases of the types of tumors found in the study, of which eight were non-Hodgkin type and three were Hodgkin's. Other types of tumors have also been found, such as carcinoma, thymoma and teratoma.

Regarding the location of the tumor in the mediastinum, the study demonstrated that fourteen were in the anterosuperior region, followed by the middle and posterior regions. The proportion-comparison test was significant with respect to the diagnosis and tumor site ( $p$-value $<0.001$ and $=0.004$, respectively), according to Table 1.

In the analysis of the vocal parameters, the lesions that occupied the anterior/ antero-superior region presented higher averages of the score in the following parameters: General voice deviation, roughness, breathiness and loudness. For tension and pitch there were higher means in the group of subjects with tumors located in the middle mediastinum, as Table 2.

Regarding the type of tumor, subjects with a diagnosis of lymphoma had higher mean values in the general deviation and in the roughness of the voice. Hodgkin lymphoma is responsible for the highest averages in all the parameters evaluated. Subjects with carcinoma-like tumors had worse results in tension and pitch, thymomas in breathability and other types of tumors in loudness (Table 2). There was no significant correlation in relation to the location and type of tumor. 
Table 1. Characterization of the sample and clinical profile of patients

\begin{tabular}{|c|c|c|c|}
\hline Variables & $\mathbf{N}$ & $\%$ & p-value ${ }^{1}$ \\
\hline \multicolumn{4}{|l|}{ Sex } \\
\hline Male & 10 & 47.6 & \multirow{2}{*}{0.827} \\
\hline Female & 11 & 52.4 & \\
\hline \multicolumn{4}{|l|}{ Age } \\
\hline Less than 40 & 10 & 47.6 & \multirow{4}{*}{0.827} \\
\hline 40 or more & 11 & 52.4 & \\
\hline Minimum-maximum & $20-60$ & - & \\
\hline Average \pm Standard deviation & $40.1 \pm 11.7$ & - & \\
\hline \multicolumn{4}{|l|}{ Stage of treatment } \\
\hline Not started on cancer treatment steps & 7 & 33.3 & \multirow{3}{*}{0.102} \\
\hline In cancer treatment & 11 & 52.4 & \\
\hline After cancer treatment & 3 & 14.3 & \\
\hline \multicolumn{4}{|l|}{ Type of treatment } \\
\hline Surgical & 5 & 23.8 & \multirow{4}{*}{0.644} \\
\hline CHT & 6 & 28.6 & \\
\hline RT & 3 & 14.3 & \\
\hline $\mathrm{CHT}+\mathrm{RT}$ & 7 & 33.3 & \\
\hline \multicolumn{4}{|l|}{ Type of tumor } \\
\hline Lymphoma & 11 & 52.4 & \multirow{5}{*}{-} \\
\hline Carcinoma & 4 & 19.0 & \\
\hline Thymoma & 3 & 14.3 & \\
\hline Teratomas & 1 & 4.8 & \\
\hline Others & 2 & 9.5 & \\
\hline \multicolumn{4}{|l|}{ Type of Lymphoma } \\
\hline Hodgkin & 3 & 27.3 & \multirow{2}{*}{0.132} \\
\hline Non Hodgkin & 8 & 72.7 & \\
\hline \multicolumn{4}{|l|}{ Location of tumor in the Mediastinum } \\
\hline Anterior / antero-superior & 14 & 66.7 & \multirow{3}{*}{0.004} \\
\hline Medium & 5 & 23.8 & \\
\hline Posterior & 2 & 9.5 & \\
\hline
\end{tabular}

${ }_{1}^{1}$ p-value Chi-square test for proportion comparison (if p-value $<0.05$ the percentage of factor levels differ). CHT: Chemotherapy; RT: Radiotherapy. \% percentage. 
Table 2. Mean and standard deviation of vocal performance parameters according to the clinical profile of patients with mediastinal tumors

\begin{tabular}{|c|c|c|c|c|c|c|}
\hline \multirow{2}{*}{ Clynical profile } & \multicolumn{6}{|c|}{ PERCEPTIVE ANALYSIS OF HEARING OF THE VOICE (CAPE-V) } \\
\hline & GG & RU & SO & TE & PITCH & LOUDNESS \\
\hline \multicolumn{7}{|l|}{ Diagnosis } \\
\hline $\begin{array}{l}\text { TU is located only in the } \\
\text { mediastinum }\end{array}$ & $56.9 \pm 24.1$ & $50.9 \pm 18.3$ & $45.6 \pm 26.2$ & $17.2 \pm 6.1$ & $53.9 \pm 16.3$ & $41.2 \pm 19.9$ \\
\hline $\begin{array}{l}\text { Second diagnosis of mediastinal } \\
\text { cancer }\end{array}$ & 42.0 & 40.0 & 47.0 & 22.0 & 60.0 & 11.0 \\
\hline $\begin{array}{l}\text { Metastasis from the primary } \\
\text { tumor to the mediastinum }\end{array}$ & $52.8 \pm 17.7$ & $52.8 \pm 16.3$ & $36.8 \pm 28.2$ & $16.5 \pm 7.3$ & $57.2 \pm 29.0$ & $69.2 \pm 3.3$ \\
\hline$p$-value & $0.801^{3}$ & $0.818^{3}$ & $0.834^{3}$ & $0.735^{3}$ & $0.710^{4}$ & $0.013^{3}$ \\
\hline \multicolumn{7}{|l|}{ Type of tumor } \\
\hline Lynphoma & $62.8 \pm 20.1$ & $57.2 \pm 17.6$ & $47.2 \pm 25.3$ & $18.0 \pm 6.7$ & $52.5 \pm 19.0$ & $36.6 \pm 19.9$ \\
\hline Carcinoma & $58.2 \pm 18.7$ & $43.8 \pm 5.7$ & $42.8 \pm 31.9$ & $19.2 \pm 6.4$ & $66.0 \pm 13.9$ & $64.8 \pm 5.6$ \\
\hline Thymoma & $55.3 \pm 20.6$ & $55.3 \pm 19.3$ & $58.7 \pm 17.7$ & $17.0 \pm 4.6$ & $59.7 \pm 4.5$ & $45.0 \pm 29.9$ \\
\hline Teratomas & 14.0 & 24.0 & 27.0 & 17.0 & 14.0 & 20.0 \\
\hline Others & $29.5 \pm 17.7$ & $35.5 \pm 12.0$ & $15.0 \pm 11.3$ & $10.0 \pm 1.4$ & $58.5 \pm 4.9$ & $65.5 \pm 7.8$ \\
\hline p-value & $0.096^{3}$ & $0.167^{3}$ & $0.404^{3}$ & $0.524^{3}$ & $0.495^{4}$ & $0.078^{3}$ \\
\hline \multicolumn{7}{|l|}{ Type of Lymphoma } \\
\hline Hodgkin & $68.0 \pm 23.5$ & $62.0 \pm 21.0$ & $59.7 \pm 23.9$ & $18.3 \pm 6.5$ & $61.7 \pm 6.4$ & $45.3 \pm 23.8$ \\
\hline Non Hodgkin & $60.9 \pm 20.0$ & $55.4 \pm 17.4$ & $42.5 \pm 25.7$ & $17.9 \pm 7.2$ & $49.1 \pm 21.3$ & $33.4 \pm 19.0$ \\
\hline$p$-value & $0.626^{1}$ & $0.605^{1}$ & $0.343^{1}$ & $0.925^{1}$ & $0.474^{2}$ & $0.403^{1}$ \\
\hline \multicolumn{7}{|c|}{ Location of the tumor in the mediastinum } \\
\hline Anterior / antero-superior & $61.4 \pm 23.7$ & $56.4 \pm 18.3$ & $50.6 \pm 28.0$ & $17.3 \pm 5.7$ & $53.2 \pm 16.5$ & $50.9 \pm 19.3$ \\
\hline Medium & $47.2 \pm 4.4$ & $42.2 \pm 1.3$ & $29.8 \pm 14.2$ & $18.2 \pm 8.5$ & $67.2 \pm 11.8$ & $30.6 \pm 24.6$ \\
\hline Posterior & $33.5 \pm 27.6$ & $32.5 \pm 12.0$ & $33.0 \pm 8.5$ & $15.0 \pm 2.8$ & $35.5 \pm 30.4$ & $41.5 \pm 30.4$ \\
\hline$p$-value & $0.165^{3}$ & $0.078^{3}$ & $0.249^{3}$ & $0.836^{3}$ & $0.139^{4}$ & $0.211^{3}$ \\
\hline
\end{tabular}

${ }^{1} \mathrm{p}$-value of Student's t-test for independent samples. ${ }^{2} \mathrm{p}$-value of the Mann-Whitney test. ${ }^{3} \mathrm{p}$-value of the ANOVA test. $4 \mathrm{p}$-value of the Kruskal-wallis test. GG: General voice Deviation; RU: Roughness; BR: breathiness; TE: Tension; TU: Tumor.

In the evaluation of swallowing, according to data presented in Figure 1, it is possible to observe that the variables normal swallowing and functional swallowing represented ten subjects of the sample. In addition, ten other subjects presented discreet or moderate oropharyngeal dysphagia, and one subject presented moderate oropharyngeal dysphagia according to O'Neil classification ${ }^{15}$.

From the clinical signs of dysphagia, the study showed that three subjects presented clinical signs of aspiration and / or laryngeal penetration, with voluntary cough after food intake and noisy cervical auscultation, with improvement after adequate compensations, requiring maneuvers for complete pharyngeal clearance, as the Shaker maneuver, multiple swallowing and effort.

Of the twenty-one subjects ${ }^{7}$, presented an increase in oral transit time, broncho-aspiratory signs, without improvement with maneuvers of protection of lower airways in a single consistency, being this liquid. In addition, four other subjects presented broncho-spastic clinical signs in two consistencies, a weak cough, and supplementation with an alternative route was required. These findings correspond to discrete/moderate and moderate oropharyngeal dysphagia, respectively. 


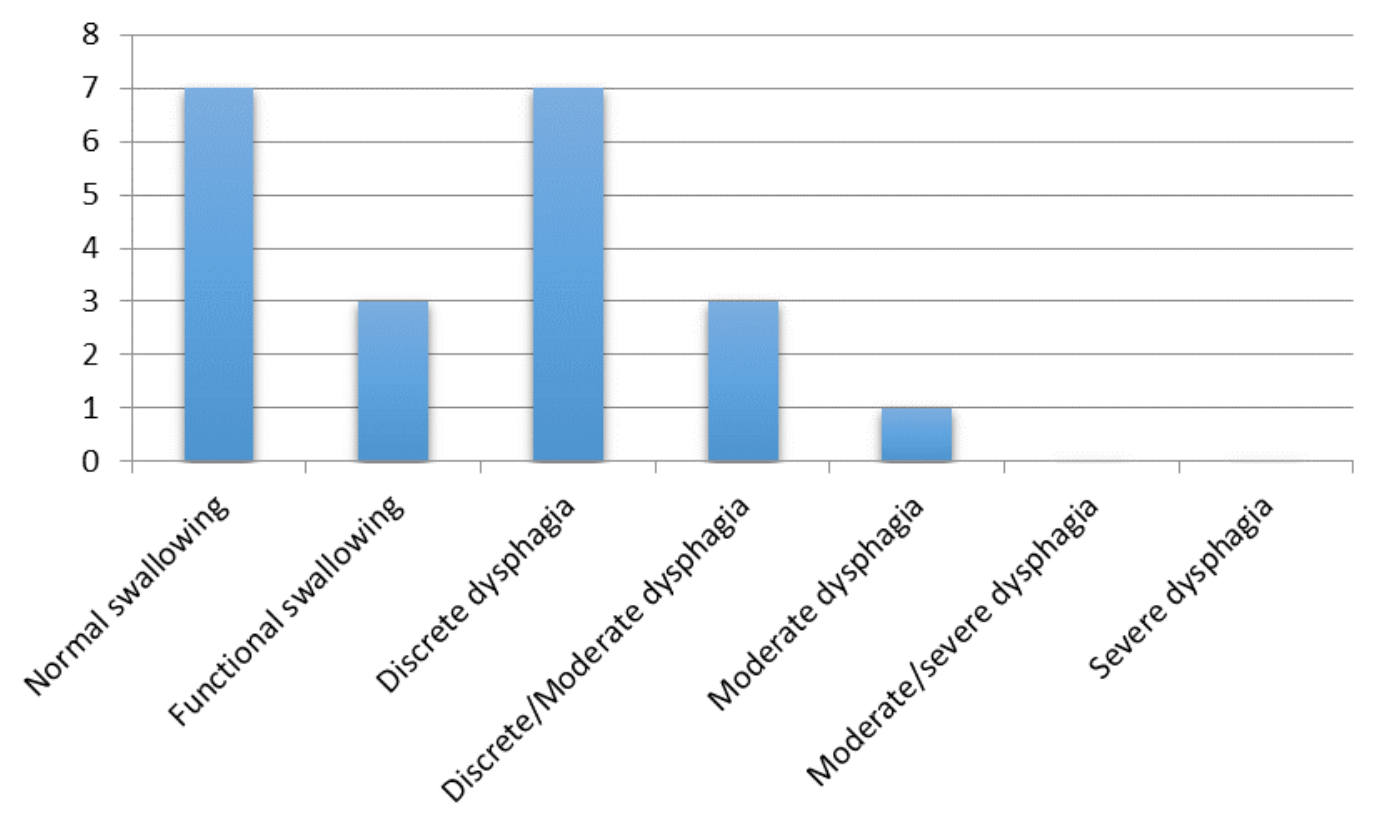

Figure 1. Distribution of the swallowing score, according to the 0'Neil swallowing scale

\section{DISCUSSION}

Tumors in the mediastinum have high rates of morbidity and mortality due to the proximity of important thoracic structures. The main symptoms that can be found are related to difficulty in breathing, cough, fever, weight loss, hoarseness, stridor, postural symptoms, superior vena cava syndrome, dysphagia and tracheal compression $^{5-7,16}$.

In this study, the prevalence of the female sex with an average age of approximately 40 years was verified, with the result that the profile of the subjects contrasts with that of other studies ${ }^{6,17,18}$, most of whom have a population of young men between 15 and 35 years of age, mainly in the second and third decades of life. The Bokemeyer study ${ }^{6}$ mentions that these tumors do not present predation by sex, with more prevalent malignant tumors in the male population.

The study with 171 patients, 100 (58\%) had tumors located in the anterosuperior mediastinum, followed by the posterior mediastinum and finally, the middle mediastinum. The most frequent tumors are thymomas, lymphomas and teratomas. Among them, lymphoma manifests as the most prevalent tumor in the study, especially in tumors in the antero-superior and middle regions ${ }^{4}$.

These findings corroborate partially with the research developed, and it was verified that there was a significant prevalence of tumors located in the anterosuperior mediastinum, and the main types of tumors present were lymphomas, carcinoma, thymomas and teratomas, respectively. Among the lymphomas, the most prevalent was non-Hodgkin type.

Due to differences in the clinical staging of each histological type present in the research, it was not possible to classify the type of treatment according to the tumor stage.

According to the vocal analysis performed in the study, they found worse vocal results in tumors located only in the mediastinum, in the lymphomas (mainly of the Hodgkin's type) and in the tumors located in the anterosuperior mediastinum. In general, the decrease in vocal performance or vocal deviation intensity presented by the study subjects may reflect on laryngeal physiology, decreasing the efficient performance of their main functions (respiration, phonation and protection of the lower airways) $)^{9,10}$.

The roughness parameter in the voice is related to the aperiodic energy in the vocal folds and may be related to roughness and / or hoarseness. In this study, moderate degree deviation in the roughness $(50.71 \mathrm{~mm})$ was registered, hoarseness being one of the symptoms most cited in the studies of patients with tumors located in the mediastinum ${ }^{7,16}$. Despite this, this parameter was not evaluated from any specific protocol in the studies presented.

With respect to the breathiness, it was evidenced that tumors in the mediastinum of the antero-superior region and lymphomas of the type Hodgkin presented worse results in this vocal parameter. This data is an 
important parameter in the evaluation of signs that functionally compromise swallowing. This occurs because the protection of the airways depends on the balance of indirect mechanisms, among them glottis competence $^{19}$.

From the clinical signs of dysphagia presented, it can be seen that fourteen subjects in the study had some degree of dysphagia, from functional swallowing to moderate dysphagia according to O'Neil scale ${ }^{15}$. With this, it can be suggested that the presence of breathy voice may be related to the prevalence of dysphagia in the studied group.

Reflex cough during and after swallowing is the classic mechanism of defense of oropharyngeal dysphagia ${ }^{20,21}$. In the study, it was possible to identify the frequency of this symptom in ten study subjects. This indicator is the existence of laryngeal sensitivity and the ability to clean airways. Despite this, its presence does not mean total clearance of the airway content, showing as the main alteration of the dysphagia of this study.

Compared to the coughing mechanism, the study presented alterations in cervical auscultation in fourteen subjects of the sample, being an important practice reported in the literature during speech therapy and it is shown as an effective tool to complement the evaluation of swallowing ${ }^{22,23}$.

The dysphagic condition is an important risk factor for increased hospitalization and increased mortality. Being identified as an area of important research, due to the negative influence on the patient's clinical prognosis ${ }^{23-25}$.

In this sense, no other specific studies related to voice and swallowing were found to confront with the data of this research. However, it can be seen that the literature reports the presence of dysphagia in tumors in the mediastinum only due to the involvement of the esophageal region ${ }^{5-8}$ and hoarseness due to bilateral bronchial compression?. The findings found in this study, becoming important for the use of objective instruments in research and / or clinical evaluations.

\section{CONCLUSION}

Through the present research, it is possible to identify that patients with tumors located in the mediastinum present potential risks for voice and swallowing disorders.

The study showed an increased risk for dysphonia, especially in patients with a diagnosis of lymphoma, mainly of the Hodgkin type, in addition to carcinomas and thymomas. This risk is also related to the location of the tumor in the mediastinum, being the anterosuperior region with worse results in the voice.

As for dysphagia, more than half of the study population presented some degree of this alteration, predominantly mild dysphagia and mild to moderate dysphagia, respectively.

Thus, it is suggested that new studies on this subject be carried with the use of objective instruments, in order to evidence the characterization of the subjects submitted to the treatment of tumors in the mediastinum and the relation of anatomophosphological changes, strengthening the investigation process of the functions of voice and swallowing.

These results are presented as important indicators for the direction of new practices/care, allowing for increasing the knowledge of the multi-professional team in the early identification of the possible speechlanguage disorders, thus, increasing the quality of the care and reducing the risks associated with worsening of the prognosis to these subjects, during the therapeutic process.

\section{REFERENCES}

1. Figueiredo E, Monteiro M, Ferreira A. Tratado de oncologia. Revinter. 1a Edição; 2013.

2. Oliveira HE, Oliveira $\mathrm{CH}$, Pietro D. Associated factors to survival of patients with mediastinal tumors. Arq. Catarin Med. 2017;46(3):2-16.

3. Dubashi B, Cyriac S, Tenali SG. Clinicopathological analysis and outcome of primary mediastinal malignancies - a report of 91 cases from a single institute. Ann Thorac Med. 2009;4(3):140-2.

4. Bastos $P$, Magalhães $A$, Fernandes $G$, Cruz MR, Saleiro $S$, Gonçalves $L$ et al. Cistos e tumores primários do mediastino. Revista Portuguesa de Pneumología. 2007;13(5):659-73.

5. Moraes RM, Tavares MRF, Vita AG, Junior CB, Sellera CAC, Ribeiro AT. Symptomatic thoracic mass in young adult: giant mediastinal teratoma. Case report. Rev Bras Clin Med. 2011;9(2):154-6.

6. Bokemeyer C, Nichols CR, Droz JP. Extragonadal germ cell tumors of the mediastinum and retroperitoneum: results from an international analysis. J Clin Oncol. 2002;20(7):1864-73.

7. Serrano AE, Arostigue AD, Crespo EC, Martínez VL. Enfermedad de Hodgkin primitiva de mediastino. Reporte de un caso. Archivo Médico de Camagüey. 2007;11(5):1-7. 
8. Aguirre MB, Pérez GR. Hodgkin pulmonar bilateral. Rev. Clin Esp. 2002;176(5):262-4.

9. Lopes LW, Silva HF, Evangelista DS, Silva JD, Simões LB, Costa e Silva PO et al . Relationship between vocal symptoms, severity of voice disorders, and laryngeal diagnosis in patients with voice disorders. CoDAS. 2016; 28(4):439-45.

10. Cohen SM, Pitman MJ, Noordzij JP, Courey M. Management of dysphonic patients by otolaryngologists. Otolaryngol Head Neck Surg. 2012;147(2):289-94.

11. Bordon A, Bokhari R, Sperry J, Testa D, Feinstein A, Ghaemmaghami V. Swallowing dysfunction after prolonged intubation: analysis of risk factors in trauma patients. Am J Surg. 2011;202(6):679-82.

12. Brown CVR, Heji K, Mandaville AD, Chaney PE, Stevenson G, Smith C. Swallowing dysfunction after mechanical ventilation in trauma patients. J Crit Care. 2011;26(1):9-13.

13. Behlau M. Consensus auditory-perceptual evaluation of voice (CAPE-V), ASHA 2003. Rev Soc Bras Fonoaudiologia. 2004;9(3):187-9.

14. Menezes MHM. Análise perceptivo-auditiva e acústica da voz relacionada ao tempo de execução do exercício de vibração sonorizada de língua em mulheres com nódulos vocais [Tese]. São Paulo (SP): Faculdade Medicina da Universidade de São Paulo; 2010.

15. O'Neil KH, Purdy M, Falk J, Gallo L. The dysphagia outcome and severity scale. Dysphagia. 1999;14(3):139-45.

16. Rajagopalan S, Harbott M, Ortiz J, Bandi V. Manejo anestésico de grande massa mediastinal para colocação de stent traqueal. Rev Bras Anestesiol. 2016;66(2):215-8.

17. Macchiarini P, Ostertag $\quad H$. Uncommon primary mediastinal tumours. Lancet Oncol. 2004;5(2):107-18.

18. Moran CA, Suster S, Koss MN. Primary germ cell tumors of the mediastinum: III. Yolk sac tumor, embryonal carcinoma, choriocarcinoma, and combined nonteratomatous germ cell tumors of the mediastinum-a clinicopathologic and immunohistochemical study of 64 cases. Cancer. 1997;80(4):699-707.

19. Chin SC, Edelstein S, Chen CY. Using to localize side and level of vocal cord paralysis. AJR Am J Roentgenol. 2003;180(4):1165-70.

20. Padovani AR, Moraes DP, Mangili LD, Andrade CRF. Protocolo fonoaudiológico de avaliação do risco para disfagia (PARD). Rev. Soc Bras Fonoaudiol. 2007;12(3):199-205.

21. Garcia MM, Magalhaes FP, Dadalto GB, Moura MVT. Avaliação por imagem da paralisia de pregas vocais. Radiol Bras. 2009;42(5):321-6.

22. Gasparim AZ, Jurkiewicz AL, Marques JM, Santos RS, Marcelino PCO, Herrero FJ. Deglutition and cough in different degrees of Parkinson disease. Arq. Int. Otorrinolaringol. 2011;15(2):181-8.

23. Mirzakhani H, Williams JN, Mello J, Joseph S, Meyer MJ, Waak K et al. Muscle weakness predicts pharyngeal dysfunction and symptomatic aspiration in long-term ventilated patients. Anesthesiology. 2013;119(2):389-97.

24. Logemann JA. Oropharyngeal dysphagia and nutritional management. Current Opinion Clin Nutr Metabolic Care. 2007;10(5):611-4.

25. Moraes DP, Sassi FC, Mangilli LD, Zilberstein B, Andrade CR. Clinical prognostic indicators of dysphagia following prolonged orotracheal intubation in ICU patients. Crit Care. 2013;17(5):243. 